\title{
ANDALUCÍA ANTE LOS RETOS DE LA GLOBALIZACIÓN. LOS PROCESOS DE INNOVACIÓN Y SUS IMPLICACIONES EN EL DESARROLLO LOCAL
}

Rosario ASIÁN CHAVES*

\section{RESUMEN}

La innovación se ha convertido en una necesidad para competir en los mercados, especialmente en los actuales globalizados. Según la mayoría de los estudios, el desarrollo de software y Servicios Avanzados basados en las Tecnologías de la Información serán los principales nuevos recursos de empleo en los primeros años del nuevo milenio. La empresa andaluza se caracteriza por una escasa diversificación tecnológica, un reducido tamaño medio, con graves problemas para financiar los altos costes de las inversiones tecnológicas. Además, se percibe una actitud empresarial poco proclive a la modernización y muy reticente a la colaboración. Sin embargo, en los últimos años se observa una tendencia a incorporar factores que favorecen la generación de una cultura de la innovación, sustentados en gran medida en la consolidación de las NTI. En este artículo se muestran algunos de los elementos que facilitan, así como los que limitan, la difusión de la innovación y sus repercusiones en el desarrollo local. Utilizamos como estudio de caso la situación de Andalucía y su relación con los efectos derivados de los procesos de "globalización".

\section{ABSTRACT}

Innovation has become absolutely essential to compete in markets, particularly in current globalised markets. According to most studies, the development of software and Advanced Services based on Information Technologies will be the main new sources of employment in first years of the new millennium. The Andalusian enterprise is characterised by the scarce technological diversification, the small average size,

\footnotetext{
* Grupo de investigación AREA. Departamento de Economía Aplicada II. Universidad de Sevilla. rasian@us.es
} 
with strong difficulties to finance the high costs of technological investments. Furthermore, it is the perception of an entrepreneurial attitude with a scarce tendency towards modernisation and highly reticent to collaborate with other enterprises and so on. However, we can observe in this region in last years a tendency to incorporate factors that help the generation of an innovation culture, sustained to a great extent in the consolidation of NIT. In this article some of the elements that make easier, as well as those that limit the diffusion of the innovation and their repercussions in the local development are shown. We have used as example the situation of Andalusia and its relation with the effects derived from the globalisation processes.

\section{INTRODUCCIÓN}

La economía mundial en la última década del siglo XX ha experimentado el paso del modelo de desarrollo industrial al "informacional". Este último se caracteriza por el papel central de la generación y tratamiento de la información en todas las actividades productivas y de gestión. Se asiste a una valorización de los activos intangibles frente a los activos físicos, que pierden importancia frente a los primeros, y a una consideración generalizada del conocimiento como factor productivo.

Con la globalización tiene lugar un resurgimiento del espacio regional como ámbito relevante para la toma de decisiones. Realmente, en palabras de Sassen (1998), las que aumentan su poder político son determinadas regiones "estratégicas". En el plano de la producción cada vez más actividades se organizan a escala planetaria, en redes de unidades de negocio conectadas entre sí, utilizan Internet ${ }^{1}$ como vehículo principal para la conexión de personas dentro de cada empresa, para la conexión entre diferentes empresas o para la de éstas con los consumidores.

Andalucía, como el resto de las economías de nuestro entorno, tiene el reto de encontrar su espacio en un mundo crecientemente interconectado, en el que la innovación se ha convertido en una necesidad para competir en los actuales mercados globalizados. Las Nuevas Tecnologías, especialmente las Tecnologías de la Información y la Comunicación (TIC), constituyen el elemento básico para conseguir la autonomía suficiente para gestionar nuestro propio futuro.

Andalucía es una región pobre en términos europeos (Hirsch, 2001), en la que se unen una serie de elementos que limitan la difusión de la innovación-escasa diversificación tecnológica; reducido tamaño medio de la empresa andaluza; se aprecia una actitud empresarial poco proclive a la modernización y con reticencias

\footnotetext{
1. Sin Internet no se podría funcionar en red y controlar procesos de producción y mercado de tamaño planetario. Para que ello sea posible es necesario contar, además de con una infraestructura tecnológica altamente desarrollada, con una gran cantidad de capital humano y de capital social de alta calidad.
} 
a la hora de colaborar con universidades y centros de investigación y, aún más, con otras empresas $^{2}-$.

No obstante, en Andalucía se manifiesta una tendencia en estos últimos años a incorporar elementos que favorecen que se genere una cultura de la innovación. Estos elementos se sustentan en gran medida en la consolidación de las Nuevas Tecnologías de la Información: actuaciones en materia de educación y formación profesional continuada, creación de redes de cooperación empresarial, intercambio de experiencias, etc. En esta región existen ciertos elementos que facilitan los procesos de innovación tecnológica, como las infraestructuras de telecomunicaciones, los centros de innovación empresarial, los parques tecnológicos, etc.

En el presente trabajo pretendemos hacer una revisión de aquellos elementos que intervienen en los procesos de innovación, centrándonos principalmente en la innovación en servicios, y más específicamente en el sector de servicios avanzados a las empresas (SAE). Antes de analizar los elementos que influyen en la generación de la innovación en Andalucía, empezamos con un breve repaso a la evolución y situación actual de la estructura económica y social de la región. Seguidamente, analizamos algunos indicadores que informan sobre el desarrollo de la Sociedad de la Información en Andalucía, lo cual sirve de referencia para tener una idea de los efectos económicos y sociales del uso de las TIC. Para el análisis empírico, nos hemos basado, además de en los datos estadísticos existentes en los elementos que componen el sistema de innovación y nuevas tecnologías, en los datos que poseemos del sector de SAE en la región andaluza ${ }^{3}$.

\section{ESTRUCTURA ECONÓMICA Y SOCIAL DE ANDALUCÍA}

La situación socioeconómica actual de Andalucía no sólo depende de su peculiar posición geoestratégica, o de factores puramente económicos, sino que es el resultado de un largo proceso histórico, en el que el papel subordinado que le ha sido adjudicado a nuestra región en el desarrollo desigual llevado a cabo en nuestro país durante las décadas de los sesenta y setenta (Delgado, 1981; Román, 1987), la trasformación político-administrativa del Estado Español derivada de la creación del Estado de las Autonomías y la integración de España en la Unión Europea, determinan fuertemente la posición actual de nuestra región.

Andalucía ocupa la primera posición, de las 17 regiones que componen el Estado Español, en términos de población (con más de 7 millones de habitantes, representa

2. A este respecto, y por lo que se refiere a las empresas oferentes de Servicios Avanzados a las Empresas en Andalucía, puede consultarse Asián (2000).

3. Nos referimos a los datos que obtuvimos a través del desarrollo de entrevistas y encuestas en empresas del sector, para la realización de la tesis doctoral (Asián, 2002). 
el $18 \%$ de la población española, siendo a su vez la tercera región más poblada de la Unión Europea), y la segunda en términos de superficie (17\%).

Por lo que se refiere a la evolución de esta región en las dos últimas décadas, respecto al capital humano, según el Padrón Municipal de Habitantes de 1996, la población andaluza ha crecido desde 1981 a 1996 en 793.888 personas, lo que supone, en términos relativos, un crecimiento acumulado del $12{ }^{\prime} 3 \%^{4}$, concentrando el $40 \%$ de todo el aumento de la población española ${ }^{5}$.

A su vez, en el período mencionado se aprecia un progresivo envejecimiento de la población andaluza, así como un extraordinario dinamismo del grupo en edad de trabajar. Sin embargo, la población andaluza es más joven que la española (Camacho, 2001). Este mayor dinamismo y juventud de la población andaluza supone un aumento de la capacidad productiva de nuestra economía, pero a su vez, ejerce una notable presión sobre el mercado de trabajo. Según la EPA, la población en edad de trabajar se incrementó en un 29'5\% desde 1980 a 1997, superando el aumento medio nacional, que fue del 20 ' $5 \%$.

En cuanto al empleo, en la década de los 90 pueden distinguirse dos subperíodos. Hasta 1994 se produce un descenso en el número de efectivos laborales tanto en Andalucía, como en España, y a partir de 1995 comienza a ascender la cifra, como puede apreciarse en el gráfico 1. Un aspecto importante es el aumento de la participación del empleo femenino a lo largo de toda la década (en Andalucía se pasa del $23^{\prime} 92 \%$ al $\left.31^{\prime} 02 \%\right)$.

La economía andaluza, al igual que las economías del entorno de la Unión Europea, se ha caracterizado por el cambio en el comportamiento sectorial de la producción y el empleo en las últimas décadas, de manera que ambas variables se han ido concentrando principalmente en los Servicios ${ }^{6}$. Según los últimos datos estadísticos, nuestra economía puede catalogarse como una "economía de servicios", ya que este sector aporta más del $50 \%$ al VAB y al empleo regional. La aportación al empleo ha pasado del 26'0\% en 1955 al 63'3\% en 1999, como puede observarse en el cuadro 1 .

4. Más del doble del registrado a escala nacional -5,3\%-

5. Además del mayor crecimiento vegetativo de la población andaluza respecto a la española, se observa también un cambio de sentido en el flujo migratorio.

6. Por lo que se refiere a la estructura porcentual del empleo, en Andalucía, al igual que en las regiones que se pueden considerar "centrales" en nuestro país, predomina ampliamente el empleo terciario, con un aumento espectacular en los últimos años. Sin embargo, aún permanece un amplio porcentaje de empleo agrario, en comparación con estas otras regiones y con la media española, mientras que el porcentaje del empleo industrial es de menos de la mitad del de Cataluña o del País Vasco. 


\section{GRÁFICO 1. \\ EFECTIVOS LABORALES POR SEXO EN ANDALUCÍA Y ESPAÑA. AÑos 1991-1999. (MILES)}

ANDALUCÍA

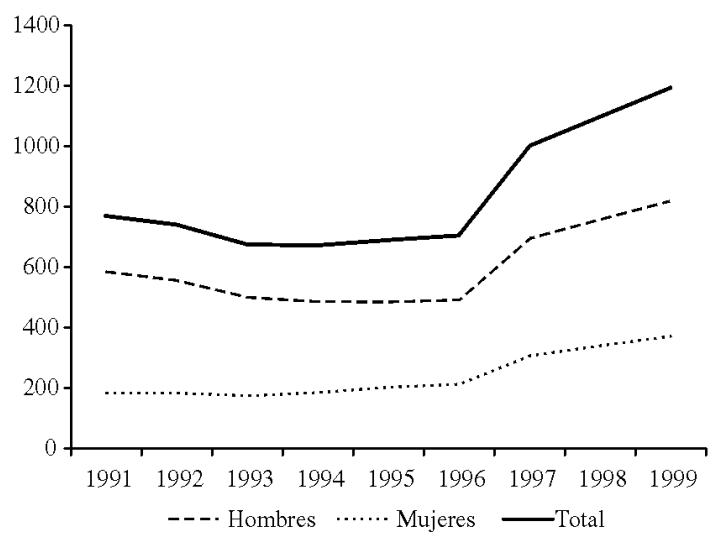

ESPAÑA

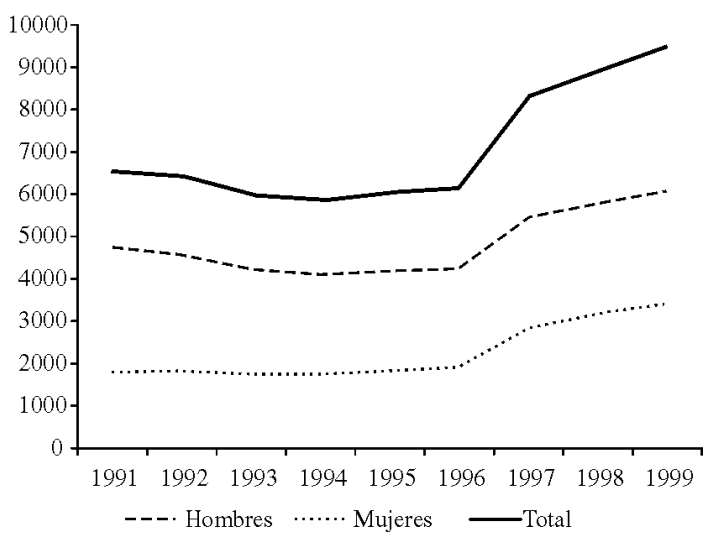

Fuente: Ministerio de Trabajo y Asuntos Sociales. Encuesta de Coyuntura Laboral.

\section{CUADRO 1.}

OCUPADOS POR SECTORES EN \% SOBRE EL TOTAL EN ESPAÑA Y ANDALUCÍA

\begin{tabular}{|c|c|c|c|c|c|c|c|c|}
\cline { 2 - 9 } \multicolumn{1}{c|}{} & \multicolumn{4}{c|}{ España } & \multicolumn{4}{c|}{ Andalucía } \\
\hline Año & $\begin{array}{c}\text { Agri- } \\
\text { cultura }\end{array}$ & Industria & $\begin{array}{c}\text { Construc- } \\
\text { ción }\end{array}$ & Servicios & $\begin{array}{c}\text { Agri- } \\
\text { cultura }\end{array}$ & Industria & $\begin{array}{c}\text { Construc- } \\
\text { ción }\end{array}$ & Servicios \\
\hline 1955 & 461 & 18,0 & 6,5 & 29,4 & 55,9 & 11,5 & 6,6 & 260 \\
\hline 1965 & 339 & 22,8 & 8,0 & 35,3 & 43,8 & 14,4 & 8,2 & 336 \\
\hline 1975 & 232 & 24,4 & 9,8 & 42,6 & 31,1 & 16,0 & 9,6 & 433 \\
\hline 1985 & 157 & 23,4 & 7,5 & 53,4 & 21,5 & 15,3 & 8,3 & 550 \\
\hline 1999 & 81 & 19,0 & 9,2 & 63,6 & 14,7 & 11,9 & 10,1 & 633 \\
\hline
\end{tabular}

Fuente: Renta Nacional de España y su distribución provincial (BBV). Elaboración propia.

La tendencia ascendente del empleo en los Servicios no se comporta de la misma manera en las distintas actividades de Servicios. El crecimiento más importante se produce en los Servicios Públicos, seguidos de Crédito y Seguros, Servicios Comerciales y Hostelería y Restaurantes (Asián, 2002). 
CUADRO 2. ÍNDICES DE ESPECIALIZACIÓN POR RAMAS DE ACTIVIDADEN ANDALUCÍA, 1961-1995

\begin{tabular}{|c|c|c|c|c|c|c|}
\hline & 1961 & 1967 & 1973 & 1981 & 1987 & 1995 \\
\hline Agricultura y Silvicultura & 1,25 & 1,27 & 1,33 & 1,40 & 1,32 & 1,73 \\
\hline Pesca Marítima & 1,60 & 1,50 & 1,37 & 1,47 & 1,50 & 1,29 \\
\hline Agricultura y Pesca & 1,26 & 1,28 & 1,33 & 1,41 & 1,33 & 1,69 \\
\hline Productos Energéticos y Agua & 0,46 & 0,53 & 0,59 & 0,61 & 0,65 & 0,74 \\
\hline Minerales y Metales & 0,86 & 0,70 & 0,56 & 0,60 & 0,70 & 0,60 \\
\hline Minerales y Productos No Metálicos & 0,75 & 0,78 & 0,87 & 0,82 & 0,81 & 0,81 \\
\hline Productos Químicos & 0,46 & 0,48 & 0,55 & 0,59 & 0,61 & 0,53 \\
\hline Productos Metálicos y Maquinaria & 0,25 & 0,30 & 0,36 & 0,39 & 0,40 & 0,41 \\
\hline Material de Transporte & 1,06 & 0,89 & 0,75 & 0,77 & 0,77 & 0,73 \\
\hline P. Alimenticios, Bebidas y Tabaco & 1,35 & 1,35 & 1,34 & 1,20 & 1,15 & 1,10 \\
\hline Textiles, Cuero y Calzado & 0,38 & 0,44 & 0,49 & 0,50 & 0,50 & 0,50 \\
\hline Papel, Art. de Papel e Impresión & 0,35 & 0,39 & 0,45 & 0,45 & 0,44 & 0,45 \\
\hline Madera, Corcho y Muebles Madera & 0,64 & 0,63 & 0,61 & 0,58 & 0,63 & 0,72 \\
\hline Caucho, Plástico y Otras Manufacturas & 0,28 & 0,29 & 0,29 & 0,39 & 0,41 & 0,43 \\
\hline Construcción e ingeniería & 0,98 & 1,03 & 1,03 & 1,13 & 1,12 & 1,06 \\
\hline Total industria sin construcción & 0,63 & 0,64 & 0,65 & 0,64 & 0,65 & 0,64 \\
\hline Industria & 0,72 & 0,75 & 0,76 & 0,76 & 0,77 & 0,77 \\
\hline Servicios destinados a la venta & 0,91 & 0,94 & 0,96 & 0,97 & 0,99 & 0,96 \\
\hline Recuperación y Reparaciones & 1,04 & 1,03 & 1,04 & 1,06 & 1,10 & 1,08 \\
\hline Servicios Comerciales & 0,85 & 0,92 & 0,96 & 1,01 & 1,02 & 1,01 \\
\hline Hostelería y Restaurantes & 1,03 & 0,99 & 1,03 & 1,05 & 1,08 & 1,08 \\
\hline Transporte y Comunicaciones & 0,84 & 0,90 & 0,94 & 0,88 & 0,92 & 0,90 \\
\hline Crédito y Seguros & 0,62 & 0,70 & 0,71 & 0,77 & 0,77 & 0,81 \\
\hline Alquiler Inmuebles & 0,46 & 0,48 & 0,56 & 0,75 & 0,74 & 0,72 \\
\hline Enseñanza y Sanidad (Privada) & 0,89 & 0,93 & 0,95 & 0,91 & 0,88 & 0,90 \\
\hline Otros Servicios para Venta & 0,96 & 0,96 & 0,93 & 0,93 & 0,93 & 0,71 \\
\hline Servicio Doméstico & 1,02 & 1,05 & 1,06 & 1,10 & 1,13 & 1,15 \\
\hline Servicios Públicos & 1,04 & 1,16 & 1,16 & 1,13 & 1,21 & 1,14 \\
\hline TOTAL SERVICIOS & 0,93 & 0,97 & 0,99 & 1,01 & 1,04 & 1,00 \\
\hline
\end{tabular}

Fuente: Renta Nacional de España y su distribución provincial (BBV). Elaboración propia. 
Resumiendo, podemos decir que en todo el período de estudio la especialización productiva andaluza se encuentra en las actividades del Sector Primario, especialización que es creciente, siendo mayor en los noventa que en las décadas anteriores. En las actividades manufactureras, Andalucía sólo presenta especialización en las relacionadas con el Sector Agroalimentario (Productos alimenticios, bebidas y tabaco), aunque ésta decrece a lo largo del período considerado. También existe una leve especialización en las actividades de Construcción.

Por último, la especialización en el Sector Servicios se debe fundamentalmente a la especialización en los Servicios públicos. Dentro de los servicios a la venta, los que presentan índices superiores a 1 son Recuperación y reparaciones y Servicios domésticos, que aumentan su especialización, y Hostelería y restauración, que la mantiene prácticamente igual. Servicios comerciales se sitúan por encima del 1 a partir de los ochenta. Aumenta la especialización en Crédito y seguros y Alquiler de inmuebles, pero sin estar cerca del 1. Sin embargo, disminuye en Otros servicios a la venta, dentro de los que se encuentran los Servicios a las Empresas.

Por tanto, como hemos comentado, la economía andaluza, tanto en términos de empleo como de producción, ha experimentado un importante avance del Sector Servicios, que ha pasado a consolidarse como primer sector económico tanto a escala regional como provincial. Sin embargo, si descendemos al ámbito provincial observamos diferencias significativas.

Para el caso de la producción, la provincia más terciarizada con diferencia del resto es Málaga -que concentra un $75^{\prime}$ ' \% de su VAB en los Servicios-, seguida de Sevilla y Granada, todas ellas con valores por encima de la media andaluza. Todas las provincias tienen porcentajes superiores al 55\% del VAB en los Servicios, y menos Almería, Huelva y Jaén, las otras cinco provincias poseen valores superiores al 60\%. Pero existen diferencias entre las provincias con respecto a las distintas ramas de Servicios ${ }^{7}$.

Las estructuras sectoriales del empleo ${ }^{8}$ por provincias presentan apreciables contrastes entre ellas y con respecto a lo que sucede en el ámbito nacional. Así, junto a estructuras muy terciarizadas, como es el caso de Málaga -con un porcentaje de empleo terciario del $72^{\prime} 6 \%$ frente al $63^{\prime} 6 \%$ nacional-, en otras provincias la Agricultura posee todavía un peso muy elevado, como en Almería (25’2\%), en Jaén (20’3\%), Huelva (20’2\%) y Córdoba (17’4\%), todas con niveles de empleo en los Servicios por debajo de la media nacional.

7. Para ver estas diferencias puede consultarse Asián (2002), capítulo 4.

8. El peso del empleo agrario es superior a la media española del 8' $1 \%$ en todas las provincias andaluzas, mientras que el empleo industrial no alcanza el valor nacional, ni en el ámbito regional ni en alguna de las provincias. Las ocho provincias andaluzas, aunque con las diferencias mencionadas, presentan porcentajes de empleo en el Sector Servicios superiores al 50\%, siendo superior a la media regional y nacional en Cádiz, Málaga y Sevilla. 


\section{ELEMENTOS QUE INFLUYEN EN LA GENERACIÓN DE PROCESOS DE INNOVACIÓN EN ANDALUCÍA}

La progresiva integración de la economía andaluza en su contexto más próximo -el nacional y el europeo-, la obliga a mejorar su posición para ser más competitiva, para lo que es necesario transformar su estructura productiva con el fin de articular su tejido económico para reducir su situación de dependencia. Para ello, además de aumentar el stock de capital físico -ampliación de las infraestructuras para mejorar su conexión con el exterior y adaptarse a la demanda de los mercados y a las fuerzas tecnológicas externas-, es necesario un mayor esfuerzo en potenciar los factores potenciales endógenos, así como los recursos intangibles que puedan generar procesos de innovación, como el capital social, cultural, humano, etc. (Alburquerque et alia, 2001).

A continuación presentamos brevemente algunos factores que favorecen una cultura de la innovación, basados en la consolidación de las TIC's -actuaciones en materia de educación y formación tendentes a mejorar la calidad del capital humano, creación de redes de cooperación empresarial, etc.-, así como algunos de los elementos que facilitan los procesos de innovación tecnológica en Andalucía -empresas del sector TIC, Parques Científicos y Tecnológicos e incubadoras de empresas-.

\subsection{Educación y formación continuada}

La innovación requiere de formas de conocimiento tácito, de difícil codificación, depositadas esencialmente en las personas. El éxito del proceso innovador depende en gran medida de la existencia de un capital humano adecuado y de su posterior incorporación al mundo laboral.

El capital humano tiene una importancia decisiva y se considera un factor esencial para atraer a las empresas a localizarse en una determinada región. La educación y la formación contribuyen a la adopción de nuevas tecnologías, teniendo también importantes efectos sobre la productividad ${ }^{9}$. Se crea así un vínculo directo entre tecnología, formación y competitividad.

En Andalucía se ha venido realizando un esfuerzo importante, desde que en 1983 obtuvo las competencias en educación, para ir superando las importantes carencias y desigualdades con respecto a la media de España ${ }^{10}$. Así, se ha reducido

9. La centralidad del factor humano en la producción, tanto industrial como, especialmente en la de servicios, origina la necesidad de una serie de servicios relacionados con la formación y capacitación de los trabajadores (Asián, 2002).

10. En 1983 la tasa de analfabetismo en Andalucía era del 11'5\% frente al 6' $15 \%$ de la media nacional. El 48'5\% de la población andaluza carecía de titulación mínima adecuada para acceder al mercado de trabajo, frente la 28 ’ $4 \%$ de media en España. (Alburquerque et alia, 2001) 
considerablemente la tasa de analfabetismo y aumentado el porcentaje de población con estudios medios y superiores.

Las cualificaciones laborales se han modificado, produciéndose un incremento del nivel de estudios de los ocupados, sobre todo en los servicios. Si observamos los datos obtenidos a partir del Censo de Población de 1991, vemos como el porcentaje de Universitarios es significativamente superior en los servicios que en el resto de ramas de actividad, situándose este porcentaje en Andalucía ligeramente por encima de la media nacional ${ }^{11}$. En contraposición, el porcentaje de analfabetos es inferior en los servicios al resto de los sectores, pero en este caso, el porcentaje para Andalucía es superior a la media nacional.

CUADRO 3.

PORCENTAJES DE ANALFABETOS Y UNIVERSITARIOS POR SECTORES, 1991

\begin{tabular}{|l|c|c|c|c|}
\cline { 2 - 5 } \multicolumn{1}{c|}{} & \multicolumn{2}{c|}{ ANDALUCÍA } & \multicolumn{2}{c|}{ ESPAÑA } \\
\cline { 2 - 5 } \multicolumn{1}{c|}{} & Analfabetos & Universitarios & Analfabetos & Universitarios \\
\hline Agricultura & 62,4 & 1,3 & 37,8 & 0,3 \\
\hline Industria & 17,0 & 5,1 & 10,8 & 7,3 \\
\hline Construcción & 32,9 & 3,2 & 21,5 & 4,3 \\
\hline Servicios & 13,9 & 19,9 & 8,1 & 19,5 \\
\hline
\end{tabular}

Fuente: INE. Censo de Población 1991.

Partiendo de la mayor presencia en el sector servicios, tomado en su conjunto, del número de universitarios, este dato hay que ponerlo en su contexto y acudir a la realidad del empleo en este sector, pues muchas veces, esta mayor presencia de universitarios no representa más que un aumento de situaciones de subempleo, en el sentido de que trabajadores universitarios están desarrollando funciones muy por debajo de sus niveles de cualificación ${ }^{12}$. Esto es debido a que el crecimiento del sistema universitario continúa sin adaptarse a los requerimientos del sistema productivo y de la sociedad, de modo que la formación que se imparte incide más en los aspectos academicistas y disciplinares que en los técnicos y profesionales.

11. Ahora bien, el sector servicios es muy heterogéneo, y concretamente en este respecto, en el sector conviven actividades altamente cualificadas -educación, investigación y desarrollo, etc.- con otras en las que se exigen pocas cualificaciones laborales -servicios domésticos, servicios personales, etc.-.

12. Es el caso de muchos cajeros en bancos y cajas de ahorro, o en numerosos comercios, que desempeñan funciones de auxiliar administrativo a pesar de sus títulos de economistas, abogados, etc. Esta situación se repite en el sector de seguros y en otras actividades comerciales, y también ha sido comprobada en el estudio realizado sobre el sector de "Servicios Avanzados a las Empresas en Andalucía" (Asián, 2002), donde se comprueba que, en muchos de los casos, las funciones de secretaria o de administrativo/a, contable, etc., la desempeñan titulados universitarios. 
Por lo que se refiere más específicamente a las capacidades humanas y técnicas de la población para, a través del uso de las TIC en la aplicación y desarrollo de nuevos conocimientos, afrontar el reto de la Sociedad de la Información y el Conocimiento, es necesario fomentar la capacitación en TIC de los recursos humanos en dos direcciones: la capacitación de especialistas técnicos de la industria de las TIC y la capacitación en el uso de las TIC para los trabajadores empleados en las restantes actividades económicas.

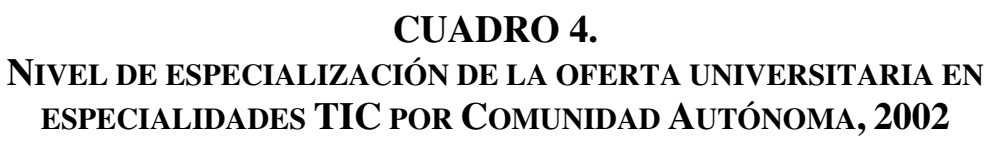

\begin{tabular}{|c|c|}
\hline Comunidad Autónoma & $\begin{array}{c}\text { Índice de especialización } \\
\text { oferta universitaria TIC }\end{array}$ \\
\hline Andalucía & 0,82 \\
\hline Aragón & 0,75 \\
\hline Asturias & 1,62 \\
\hline Baleares & 1,47 \\
\hline Canarias & 1,30 \\
\hline Cantabria & 0,86 \\
\hline Catilla-La Mancha & 0,91 \\
\hline Castilla y León & 1,32 \\
\hline Cataluña & 1,14 \\
\hline Ceuta y Melilla & 1,00 \\
\hline C. Valenciana & 1,11 \\
\hline Extremadura & 1,02 \\
\hline Galicia & 0,58 \\
\hline Madrid & 1,09 \\
\hline Murcia & 0,94 \\
\hline Navarra & 1,19 \\
\hline País Vasco & 0,90 \\
\hline Rioja & No dispone \\
\hline
\end{tabular}

Fuente: Fundación AUNA 2002, a partir de MEC (2002).

NOTA: El cálculo del índice ha sido: $\left(\mathrm{TIC}_{\mathrm{CCAA}} / \mathrm{TIC}_{\mathrm{Esp}}\right) /\left(\mathrm{TOT}_{\mathrm{CCAA}} / \mathrm{TOT}_{\mathrm{ESP}}\right)$, es decir el ratio entre la razón de la oferta en titulaciones TIC en las universidades públicas de cada CCAA y la total de España y la razón de la oferta total universitaria. Los valores superiores a 1 indican un grado de especialización en especialidades TIC en relación con el conjunto nacional. 
Andalucía es una de las regiones españolas con menor grado de especialización en la formación universitaria en TIC $^{13}$, superando tan sólo a Galicia y Aragón. Un dato significativo de la preparación de la población andaluza es la disparidad que existe entre la oferta y la demanda de titulaciones por parte de la industria. El capital humano andaluz muestra una menor orientación hacia las titulaciones de carácter técnico que las regiones consideradas como "más desarrolladas" en nuestro país (Cataluña, Madrid, País Vasco). Así, la distribución por especialidades en la formación universitaria andaluza se caracteriza por la importancia de la oferta en Ciencias Sociales (47,5\% de los alumnos matriculados) y el menor peso de las Ciencias Experimentales (11\%). Esto es debido a que el crecimiento del sistema universitario continúa sin adaptarse a los requerimientos del sistema productivo y de la sociedad, de modo que la formación que se imparte incide más en los aspectos academicistas y disciplinares que en los técnicos y profesionales

\subsection{Creación de redes de cooperación empresarial}

La formación de entornos empresariales es un factor fundamental para facilitar la difusión de la innovación. En este sentido adquiere una importancia fundamental la promoción y difusión de información estratégica, la cooperación empresarial y el fomento de redes y racimos. La Red Andalucía Innovación es un ejemplo de cooperación empresarial y uso de las nuevas tecnologías por parte de las empresas andaluzas.

Un aspecto muy importante en la construcción de redes empresariales es la utilización de las TIC's. Las empresas que actúan en Andalucía como proveedoras de acceso a Internet representan en la actualidad algo más del 8\% del resto del total nacional.

Las empresas proveedoras de acceso a Internet en Andalucía se localizan principalmente en las provincias de Málaga y Sevilla, que concentran algo más de la mitad del total de estas empresas en la región. En el extremo opuesto se encuentran las provincias de Almería, Jaén y Huelva, con 10, 5 y 2 empresas respectivamente.

13. Las titulaciones universitarias TIC incluyen las siguientes especialidades: Ingeniero Superior de Telecomunicaciones, Ingeniero en Electrónica, Ingeniero Superior de Informática, Ingeniero Técnico de Telecomunicaciones e Ingeniero Técnico de Informática. 
CUADRO 5.

NÚMERO DE EMPRESAS PROVEEDORAS DE ACCESO A INTERNET EN ESPAÑA POR CC.AA.

\begin{tabular}{|c|c|}
\hline Comunidades Autónomas & $\mathrm{N}^{\mathrm{o}}$ Empresas \\
\hline Andalucía & 151 \\
\hline Aragón & 50 \\
\hline Asturias & 33 \\
\hline Baleares & 35 \\
\hline Canarias & 29 \\
\hline Cantabria & 26 \\
\hline Catilla La Mancha & 21 \\
\hline Castilla León & 51 \\
\hline Cataluña & 389 \\
\hline Ceuta & 4 \\
\hline C. Valenciana & 150 \\
\hline Extremadura & 9 \\
\hline Galicia & 61 \\
\hline Madrid & 504 \\
\hline Melilla & 0 \\
\hline Murcia & 36 \\
\hline Navarra & 38 \\
\hline País Vasco & 75 \\
\hline Rioja & 10 \\
\hline Extranjeros & 186 \\
\hline Total & 1858 \\
\hline
\end{tabular}

Fuente: Es-NIC (www.es-nic.com). Actualizado al 13-01-03.

\subsection{Empresas del sector TIC en Andalucía}

Una de las variables a utilizar como indicador del crecimiento del sector TIC en los últimos años es el número de empresas que lo constituyen. Según los datos del DIRCE, la tasa de crecimiento interanual del número de empresas del sector TIC se ha reducido en el año 2001 respecto al 2000, posiblemente por la saturación 
del sector ${ }^{14}$. Sin embargo, la participación del número de empresa andaluzas sobre el total nacional aumenta ligeramente (del 15'7\% en 1999 al $16^{\prime} 0 \%$ en 2000 y al 16 ' $1 \%$ en 2001).

Según la base de datos de empresas del sector TIC en Andalucía utilizada por la Asociación de Empresarios de Tecnologías de la Información y Comunicación de Andalucía (ETICOM) en el "Estudio de mercado sobre el sector de informática y comunicaciones de Andalucía", en 1999 existían en nuestra región 3.064 empresas del sector TIC, de las que el $72 \%$ pertenecía al sector de informática y el resto al de comunicaciones. Estas empresas se concentran principalmente en las provincias de Sevilla (31’7\%) y Málaga (17’1\%) (Castaño y Román, 2002).

\section{FIGURA 1. \\ Número de empresas proveedoras de acceso a Internet en Andalucía por provincias}

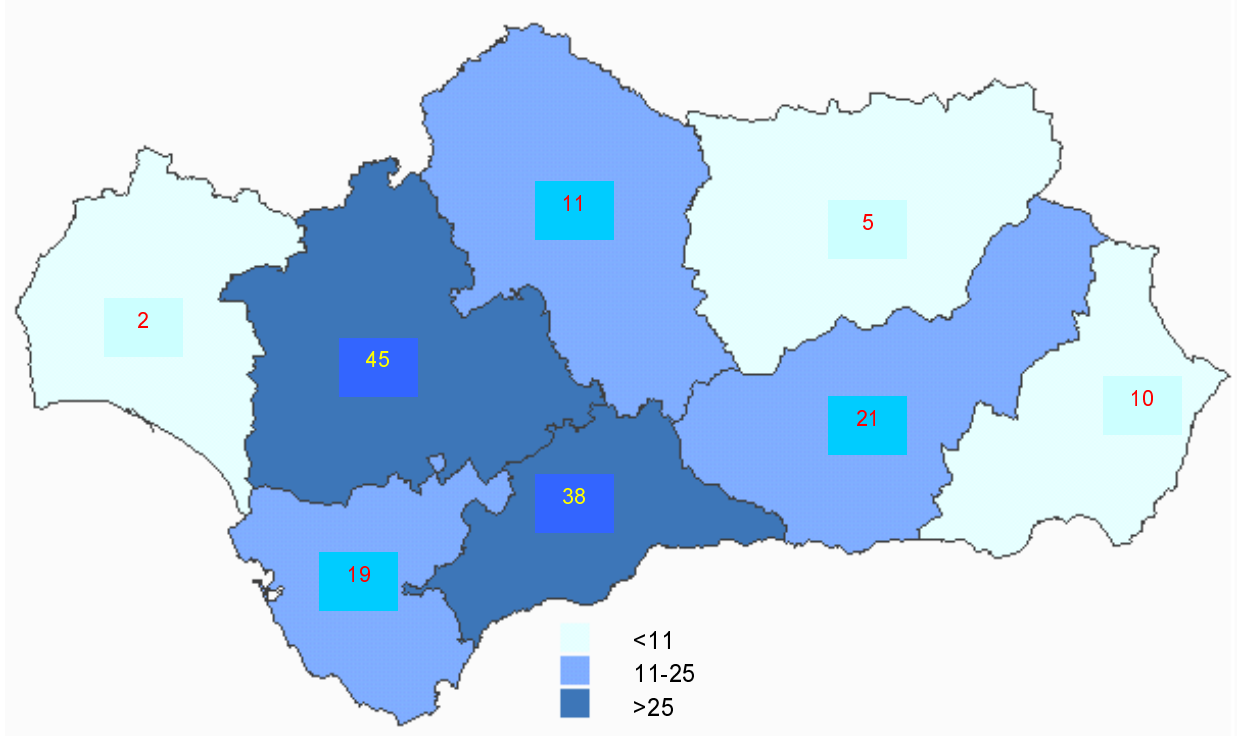

Fuente: ES-NIC (www.es-nic.com) Actualizado al 13-01-03.

14. Los subsectores considerados han sido: Comercio al por mayor y al por menor-que abarca alrededor del $90 \%$ de las empresas-, Servicios Informáticos, Fabricación de maquinarias, equipos y componentes, y Telecomunicaciones. 


\section{GRÁFICO 2. \\ EVOLUCIÓN DEL NÚMERO DE EMPRESAS DEL SECTOR TIC \\ EN ESPAÑA Y ANDALUCÍA}

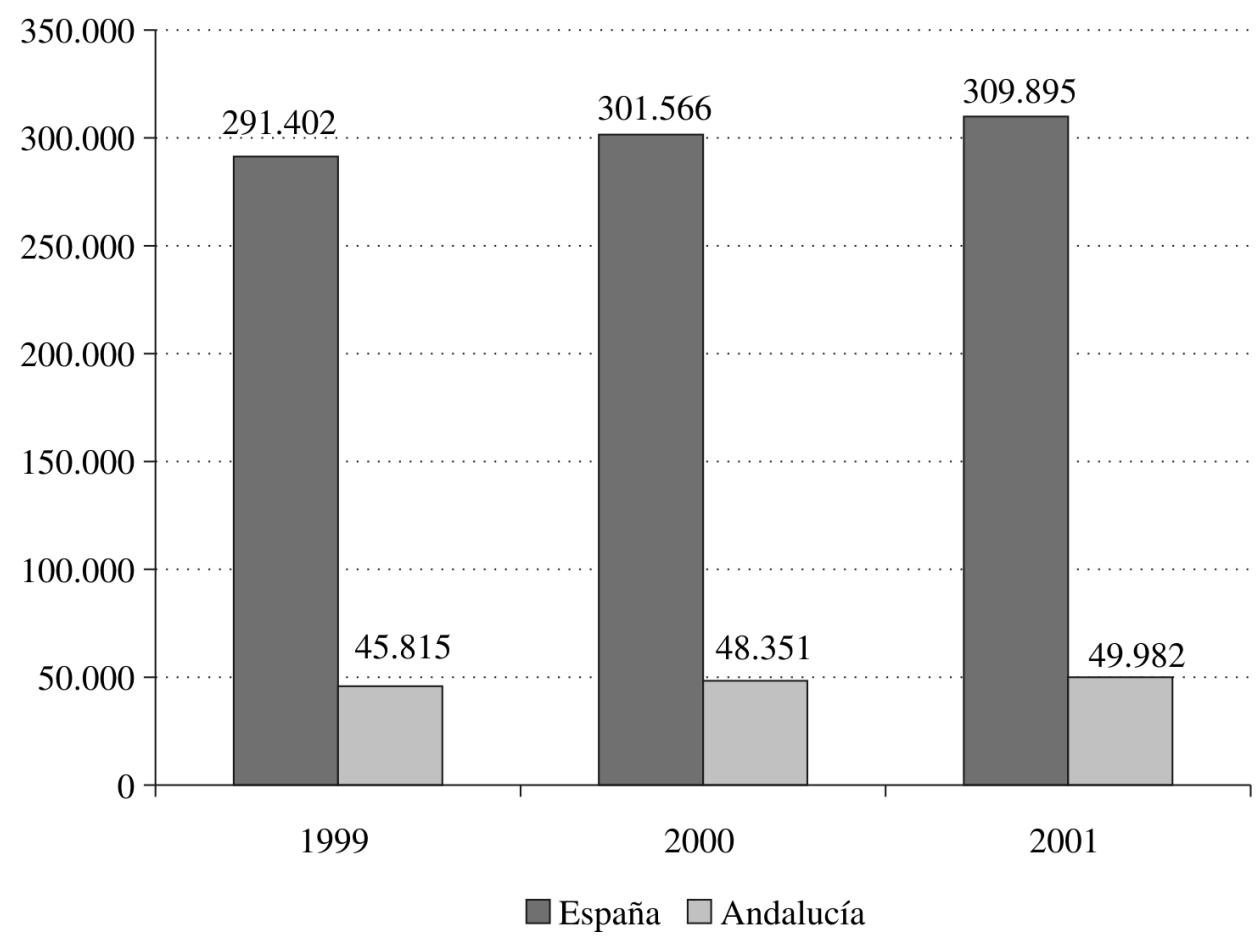

Fuente: Datos del Directorio Central de Empresas (DIRCE). Elaborado por IDR (Castaño y Román, 2002).

\subsection{Parques Científicos y Tecnológicos}

La revolución del conocimiento que ha provocado el desarrollo de las Tecnologías de la Información ha convertido a la innovación empresarial en una necesidad creciente en la economía actual. Estas Tecnologías de la Información han sido el mejor instrumento para favorecer el desarrollo empresarial y la transferencia de tecnología en los Parques Tecnológicos. La mayor parte de los centros tecnológicos en TIC en España se encuentran en el País Vasco, Cataluña, Andalucía y Castilla León. Muchos de estos centros están situados en el interior de los parques científicos y tecnológicos regionales. 
En Andalucía hemos de mencionar el papel del Parque Tecnológico de Andalucía (PTA) localizado en Málaga, y del Parque Científico y Tecnológico de La Cartuja, ubicado en Sevilla.

\section{GRÁFICO 3.}

\section{CENTROS TECNOLÓgICOS EN TIC POR CC.AA.}

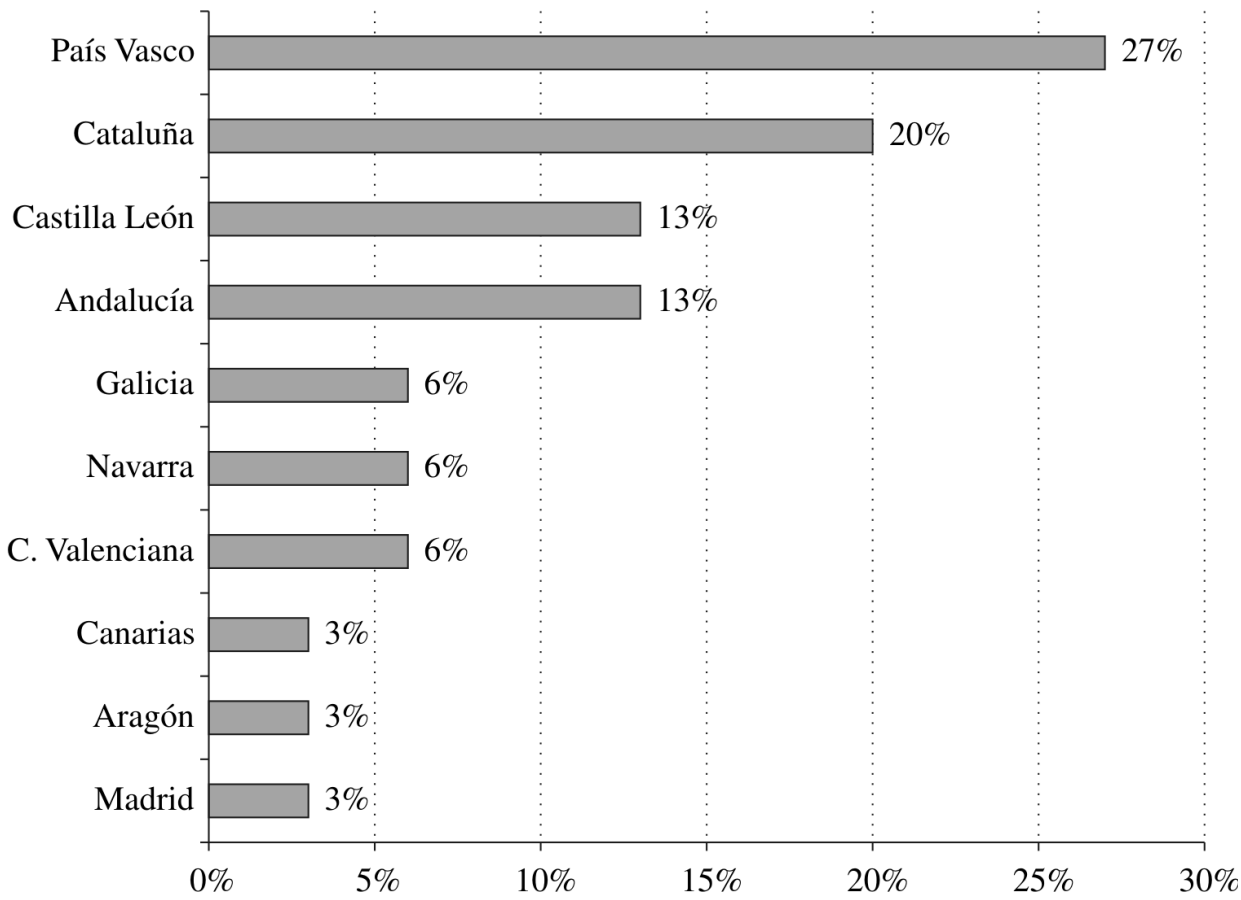

Fuente: Fundación COTEC.

El 9 de Diciembre de 1992 se inaugura el Parque Tecnológico de Andalucía(PTA), con el objetivo fundamental de conseguir que el desarrollo tecnológico pueda ayudar al desarrollo económico de la región. Actualmente cuenta con unas 200 empresas e instituciones dedicadas a la producción, la prestación de servicios avanzados y de I+D, y centros de investigación y formación, cuyas áreas de actividad preferente son el sector de las telecomunicaciones, la automatización industrial y ofimática, nuevos materiales, energías renovables y biotecnología.

El PTA está inmerso en un proyecto de teleparques que pretende potenciar y fomentar el Teletrabajo en los Parques Naturales de Andalucía para evitar la despoblación rural. El PTA ha creado el Centro Virtual de Documentación (Virtualdoc) que 
cuenta con un directorio de recursos Internet para ofrecer información especializada a las empresas del PTA en aquellas áreas en las que centra su actividad empresarial.

Además de las infraestructuras básicas, desde el PTA se ha promovido la construcción de edificios de uso múltiple, como la Sede Social -alberga a la sociedad gestora y es la sede mundial de la Asociación Internacional de Parques Tecnológicos-; Bic-Euronova -que es un centro europeo de empresas e innovación, destinado a iniciativas innovadoras e incubadora de empresas-; el Centro de Empresas (EdificioNido) - edificio de oficinas en régimen de alquiler para empresas innovadoras-; el Centro de Formación ocupacional en tecnologías de la información y telecomunicaciones; etc.

En el 2000, aproximadamente el $42 \%$ de las actividades de tecnologías avanzadas del Parque Científico y Tecnológico Cartuja' 93 eran del sector de telecomunicaciones e informática, que es también el sector que generó más actividad económica y más empleo en el parque, y con mayor número de empresas en el mismo.

CUADRO 6.

DISTRIBUCIÓN POR RAMAS DE ACTIVIDAD DE LOS SECTORES DE TECNOLOGÍAS AVANZADAS DE CARTUJA'93

\begin{tabular}{|l|c|r|r|}
\hline \multicolumn{1}{|c|}{ Tecnologías avanzadas } & Actividad económica $\left(10^{6} €\right)$ & Empleo & $\mathrm{N}^{\circ}$ empresas \\
\hline Biotecnología y agroalimentación & 7,33 & 16 & 4 \\
\hline Centros de I+D & 54,05 & 763 & 25 \\
\hline Energía & 19,86 & 151 & 3 \\
\hline Formación y docencia & 23,38 & 205 & 12 \\
\hline Ingenierías aplicadas & 73,33 & 523 & 13 \\
\hline Medio ambiente & 101,51 & 786 & 5 \\
\hline Tecnologías sanitarias & 6,89 & 105 & 3 \\
\hline Telecomunicaciones e informática & 214,90 & 2.154 & 37 \\
\hline Universidad & 12,02 & 440 & 1 \\
\hline \multicolumn{1}{|c|}{ Total } & 513,28 & 5.143 & 103 \\
\hline
\end{tabular}

Fuente: Inventario y evaluación tecnológica de las empresas y organizaciones instaladas en el Parque Científico y Tecnológico Cartuja'93. IAT, 2001.

Por lo que se refiere a las empresas de servicios avanzados, en el 2000, la mayor proporción de actividad económica se debió al sector de Medios de comunicación e información. 
CUADRO 7.

DISTRIBUCIÓN POR RAMAS DE ACTIVIDAD DE LOS SECTORES DE SERVICIOS AVANZADOS DE CARTUJA'93

\begin{tabular}{|l|c|c|c|}
\hline \multicolumn{1}{|c|}{ Tecnologías avanzadas } & Actividad económica $\left(10^{6} €\right)$ & Empleo & N $^{\mathbf{0}}$ empresas \\
\hline Asesoría empresarial & 31,46 & 337 & 12 \\
\hline Asociaciones empresariales & 10,31 & 116 & 6 \\
\hline Gestión comercial y marketing & 30,47 & 191 & 4 \\
\hline Gestión y centros de empresas & 10,16 & 117 & 6 \\
\hline $\begin{array}{l}\text { Imagen, sonido, comunicación } \\
\text { y producción }\end{array}$ & 1,86 & 281 & 2 \\
\hline $\begin{array}{l}\text { Medios de comunicación e } \\
\text { información }\end{array}$ & 50,87 & 36 & 8 \\
\hline $\begin{array}{l}\text { Representación, distribución y } \\
\text { comercialización de productos }\end{array}$ & 10,18 & 1140 & 41 \\
\hline \multicolumn{1}{|c|}{ Total } & 145,32 & & 3 \\
\hline
\end{tabular}

Fuente: Inventario y evaluación tecnológica de las empresas y organizaciones instaladas en el Parque Científico y Tecnológico Cartuja'93. IAT, 2001.

Uno de los centros tecnológicos andaluces cuya labor en TIC está siendo muy numerosa y relevante es el conformado por la Asociación de Investigación y Cooperación de Andalucía (AICIA), que desarrolla actividades de investigación industrial y ejerce un claro papel de interfaz entre la empresa y la investigación.

\section{LA INNOVACIÓN EN LOS SERVICIOS AVANZADOS A EMPRESAS EN ANDALUCÍA}

El estudio de la innovación ${ }^{15}$ en los sectores manufactureros comenzó hace varios años, sin embargo es reciente en el caso de los servicios, por lo que aún no disponemos de una doctrina generalmente conocida y aceptada.

Tradicionalmente se consideraba a los servicios como poco innovadores y que se limitaban a consumir las innovaciones producidas por las industrias manufactureras. No obstante, la evidencia reciente muestra que el sector servicios desempeña un

15. Según la naturaleza de la innovación, ésta puede ser de tres tipos: innovación tecnológica, comercial y organizativa. 
importante papel en la generación y la difusión de innovaciones ${ }^{16}$. De este modo, los análisis de prestigiosos expertos en el estudio de los servicios (Antonelli, 2000; Boden y Miles, 2000; Howells, 2002) y los informes actuales de organismos internacionales (Comisión Europea, 2002, 2003) reconocen explícitamente que los servicios desempeñan un papel importante tanto en relación con la producción como con la transmisión de conocimiento. Con ello se está haciendo referencia a los denominados servicios a empresas intensivos en conocimiento ${ }^{17}$ (KIBS, Knowledge Intensive Business Services), dentro de los cuales juegan un papel fundamental los denominados servicios avanzados a las empresas ${ }^{18}$.

Si nos centramos en el dinamismo empresarial, hemos de tener en cuenta que la innovación está en el centro del espíritu empresarial. En la mayoría de los casos, una nueva empresa nace de una actuación innovadora. Después, para continuar y crecer, la empresa debe estar permanentemente innovando, aunque la mejora técnica no es garantía del éxito. La innovación también consiste en anticiparse a las necesidades del mercado, ofrecer una calidad o unos servicios adicionales, organizar eficazmente, etc.

En el caso de las empresas de Servicios Avanzados a las Empresas, según un estudio realizado (Asián, 2002), la gran mayoría (el 75\%) de las empresas entrevistadas contempla planes de expansión, tomando éstos formas diferentes según las áreas de servicios a que pertenecen dichas empresas. Generalmente, las empresas tienden a escoger la vía de la penetración en nuevos mercados ${ }^{19}$ y la intensificación de los servicios existentes, más que la de ampliación de los servicios. Casi todas las empresas prevén la creación de empleo. Las opciones de ofrecer paquetes integrados de servicios y la de diversificar la gama, no son tenidas en cuenta por la mayoría de estas empresas. Esto significa, que las posibilidades de introducir procesos de estandarización y de aprovechar las economías de escala, no estarían consideradas dentro de las opciones de competir en unas mínimas condiciones de igualdad con las empresas externas.

Tampoco parece que las empresas de Servicios Avanzados en Andalucía vayan a adoptar, como uno de sus comportamientos empresariales de cara al futuro, la innovación en procesos y en productos (posibilidad que no ha considerado el $90 \%$ de las

\footnotetext{
16. Así, algunas de las actividades clasificadas como servicios avanzados a las empresas, como es el caso de los servicios de consultoría, no generan innovaciones por sí mismas, pero contribuyen a la difusión de la innovación a otros sectores.

17. Los denominados servicios intensivos en conocimiento incluyen las ramas 61, 62, 64-67, 70-74, 80, 85, 92, de las cuales no se puede diferenciar claramente cuáles son las actividades realmente intensivas en conocimiento y cuáles no.

18. Una clasificación más restrictiva que la de KIBS es la de servicios avanzados a las empresas, aunque tampoco es una clasificación exhaustiva, de modo que puede ser discutible si algunas de las actividades que comprende son realmente servicios avanzados e innovadores. Para ver las actividades consideradas puede consultarse en el capítulo de metodología de Asián (2002).

19. Pero, la mayoría no se refieren a la penetración en mercados exteriores, sino dentro de la propia región.
} 
empresas estudiadas), lo cual podría posibilitar la configuración de una oferta dinámica y adecuada a las necesidades que se plantean actualmente, derivadas de la norma competitiva internacional.

\section{CUADRO 8.}

PORCENTAJES DE EMPRESAS QUE CONTEMPLAN PLANES DE EXPANSIÓN POR ÁREAS DE SERVICIOS

\begin{tabular}{|l|c|c|c|c|c|c|}
\hline \multicolumn{1}{|c|}{$\begin{array}{c}\text { Áreas de } \\
\text { servicios }\end{array}$} & $\begin{array}{c}\text { Planes de } \\
\text { expansión }\end{array}$ & $\begin{array}{c}\text { Intensificación- } \\
\text { especialización }\end{array}$ & $\begin{array}{c}\text { Nuevos } \\
\text { mercados }\end{array}$ & $\begin{array}{c}\text { Mecanizar- } \\
\text { innovar }\end{array}$ & $\begin{array}{c}\text { Ampliar } \\
\text { la gama }\end{array}$ & $\begin{array}{c}\text { Paquetes } \\
\text { integrados }\end{array}$ \\
\hline $\begin{array}{l}\text { Recursos } \\
\text { Humanos }\end{array}$ & 66,7 & 22,2 & 33,3 & 44,4 & 22,2 & 22,2 \\
\hline Producción & 76,9 & 30,8 & 46,2 & 30,8 & 23,1 & 0 \\
\hline Mercados & 71,4 & 21,4 & 42,9 & 14,2 & 21,3 & 0 \\
\hline Gestión & 84,2 & 52,6 & 63,2 & 31,6 & 15,8 & 26,3 \\
\hline $\begin{array}{l}\text { Sistemas de } \\
\text { Información }\end{array}$ & 88,9 & 55,6 & 77,7 & 33,3 & 44,4 & 0 \\
\hline $\begin{array}{l}\text { Estudios y } \\
\text { Proyectos }\end{array}$ & 69,4 & 25,0 & 55,6 & 14,0 & 30,6 & 0 \\
\hline
\end{tabular}

Fuente: Elaboración propia.

La proliferación de acuerdos con otras compañías para desarrollar proyectos en común y la generación de redes empresariales, pretenden aumentar la dimensión organizativa manteniendo una dimensión técnica relativamente reducida. Aquí, como han indicado diversos autores (Alburquerque et alia, 2001), se manifiestan las debilidades del tejido productivo andaluz, al no verse compensadas las dificultades producidas por el pequeño tamaño con un planteamiento de cooperación empresarial ${ }^{20}$.

Concretamente, en un estudio realizado sobre el sector se Servicios Avanzados a las Empresas en Andalucía (Asián, 2002), se pone de manifiesto la escasa tendencia a utilizar el asociacionismo como posibilidad para las pequeñas empresas de servicios locales de alcanzar las necesarias economías de escala para poder competir con las grandes empresas de servicios. De las empresas entrevistadas, sólo un $42 \%$ declaran pertenecer a alguna red. El 36' 1\% de las empresas que dicen pertenecer a alguna red, fueron constituidas en los noventa. Coinciden con las empresas de mayor tamaño, dominando las empresas cuya actividad está relacionada de algún modo con los servicios de ingeniería (el 19\%).

20. Aunque la tendencia al asociacionismo está aumentando, a mediados de los noventa tan sólo el $25 \%$ de las empresas andaluzas tenía algún tipo de acuerdo de cooperación con otras empresas. 
Por lo que se refiere a la posibilidad de impulsar o facilitar la innovación en las empresas que utilizan los servicios avanzados propicios para ello, del estudio citado (Asián, 2002) se deduce que existe un escaso uso de estos servicios por parte de las PYMES andaluzas ${ }^{21}$, y un aún más reducido nivel de externalización de los mismos. Además, dentro de los servicios que se demandan a empresas especializadas en su provisión, los menos utilizados son los de asesoramiento en I+D, asesoramiento en logística y planificación estratégica. Sin embargo, el servicios que sí se suele demandar externamente con relativa frecuencia es el asesoramiento en comercio exterior (se externaliza su uso en un 71'4\%, aunque su porcentaje de uso sólo alcanza el 25\%).

\section{ACTUACIONES DESDE LA ADMINISTRACIÓN AUTONÓMICA}

Entre los factores que más inciden en el desarrollo de la Sociedad de la Información están las actividades puestas en marcha por la Administración Pública para promocionar y fomentar la misma. El potencial innovador de las empresas está condicionado por la preocupación existente en sus países de origen por desarrollar la actitud innovadora en la sociedad, pues el modo en que se gestionan los recursos dedicados a potenciar el sistema de ciencia y tecnología es esencial para la generación y difusión de innovaciones (Cotec, 2001). Dentro de los incentivos financieros, la tendencia reciente en España es hacia la expansión de los incentivos fiscales en detrimento de las subvenciones.

Las administraciones autonómicas también desarrollan actividades de promoción de la innovación. La importancia económica e institucional de las políticas autonómicas de innovación es muy diversa, no teniendo un marco general de referencia, ni siendo objeto de coordinación, ya que, generalmente, se han centrado en el desarrollo tecnológico industrial y se han concentrado en ayudas a las empresas y en la creación de infraestructuras de soporte a la innovación -centros y parques tecnológicos, etc.-.

Sólo recientemente se ha despertado cierta preocupación por las consecuencias económicas del cambio tecnológico, posiblemente debido a que hasta hace poco este cambio era lo suficientemente lento como para que las teorías económicas consideraran a la tecnología como un factor exógeno al que los sistemas económicos debían adaptarse de un modo u otro. La actual globalización de las relaciones ciencia-tecnología-economía está imponiendo nuevos tratamientos y exigencias a la investigación científica, al mismo tiempo que considera la innovación como fuente fundamental de progreso y riqueza económica.

21. El estudio se realizó para las empresas de la Industria Agroalimentaria, que se había manifestado como uno de los sectores principal demandante de este tipo de servicios, junto a la Administración Pública y la Construcción. 
El gobierno andaluz viene desarrollando desde 1993 una fórmula de concertación social para consensuar propuestas de políticas públicas con los principales agentes económicos y sociales. En este sentido se han venido desarrollando el Acuerdo para el Desarrollo Económico y Social de Andalucía (1993/94), el Pacto por el Empleo y la Actividad Productiva (1995/96), el Pacto por el Empleo y el Desarrollo Económico de Andalucía (1997/98) y actualmente está vigente el Acuerdo de Concertación Social de Andalucía.

Los datos de seguimiento del Pacto por el Empleo y el Desarrollo Económico de Andalucía (1997/98), ofrecen los siguientes datos sobre las políticas de fomento de la actividad empresarial: se ha inducido una inversión de la iniciativa privada de 635.523 millones de pesetas en 9.921 empresas, se han creado 29.685 empleos y se ha contribuido a mantener 109.033 empleos, para lo cual se ha realizado un gasto público de 51.544 millones de pesetas (Consejería de Economía y Hacienda, Junta de Andalucía).

Para lograr que Andalucía dé un gran salto cualitativo es preciso poner en funcionamiento un Plan Estratégico de Desarrollo Tecnológico Integral. En Andalucía, desde 1984 se comienzan a poner los cimientos de una verdadera política científica, que se concreta en la actualidad en el III Plan Andaluz de Investigación, que regula todo el proceso de generación de conocimiento en nuestra región, e implica a las Universidades y sus grupos de investigación, a los Institutos y Centros de I+D y a las Oficinas de Transferencia de Resultados de la Investigación (OTRI's).

Sin embargo, se ha puesto de manifiesto que la participación de las empresas en estos procesos es escasa. Por ello se pone en marcha el Plan Director de Innovación y Desarrollo Tecnológico (PLADIT 2001-2003), como marco global de coordinación de los distintos instrumentos y agentes involucrados en los procesos de innovación y tecnología. Este Plan Director se articula, fundamentalmente, con la creación del Centro Andaluz de Servicios Tecnológicos Aplicados (CASTA), como unidad de coordinación de la oferta de innovación y tecnología, y con la puesta en marcha de RAITEC $^{22}$ (Red Andaluza de Innovación y Tecnología) que agrupará a todos los organismos del subsistema de innovación, desarrollo tecnológico y transferencia para ofrecer un conjunto ordenado de servicios que sirvan para satisfacer las necesidades empresariales en materia de Innovación y Tecnología.

22. RAITEC ha sido creada por la Consejería de Empleo y Desarrollo Tecnológico (su origen se encuentra en el PLADIT 2001-2003) para articular la conexión de los Agentes Tecnológicos que la componen, servidores de servicios, con el tejido productivo andaluz, demandantes de servicios. La red proporciona información y conocimiento y permite además el acceso directo a los servicios ofertados por los Agentes Tecnológicos. Estos Agentes Tecnológicos son de los siguientes tipos: Parques Tecnológicos, Tecnoparques, Centros de Innovación y Tecnologías, Centro Incubadora de Empresas, Centros Europeos de Empresas e Innovación, Redes y Centros de I+D+I, Agencias de Desarrollo, etc. 
Otras iniciativas de la Consejería de Empleo y Desarrollo Tecnológico para tratar de impulsar la Sociedad de la Información y el Conocimiento (SIC) en Andalucía son la puesta en marcha del Programa Ciberpyme desarrollado en el año $2001^{23}$, Proyecto i-Arco ${ }^{24}$, Proyecto Picasso ${ }^{25}$, Proyecto Prometeo ${ }^{26}$, el portal en Internet de servicios con información para búsquedas de empleo denominado Andalucía orienta y la Iniciativa comunitaria EQUAL (2001-2004), dirigida a la eliminación de las desigualdades en el mercado laboral entre hombres y mujeres, incluyendo entre sus actuaciones el aprendizaje de las nuevas tecnologías como punto fundamental a la inserción laboral.

Dentro de la CEDT, a través del Instituto de Fomento de Andalucía (IFA), se han llevado a cabo las siguientes acciones:

Plan de Consolidación y Competitividad de la PYME (PCCP) (2001-2006) con el objetivo de introducir a las PYMES en la SI. EmprendeJoven, para el fomento de una cultura emprendedora e innovadora entre jóvenes que estudien en alguna rama de la industria, las tecnologías o los servicios y tengan un plan de empresa. Los planes se gestionan desde el Centro Andaluz de Emprendedores (CADE). Programas de Ayudas IFA. Centro de Información Tecnológica y de la Propiedad Industrial de Andalucía (CITPIA) cuya función es actuar como servicio de información y asesoramiento y contribuir al desarrollo tecnológico y de la competitividad del sector empresarial andaluz. Actúa como un centro de transferencia tecnológica.

Por otro lado, a través del Instituto Andaluz de Tecnología (IAT), se vienen desarrollando los siguientes proyectos TIC: eBIZ (formación para empresas en eBusiness), IberoVIWIN (red para la difusión de tecnologías wireless en España), MULTINET (red para la promoción del uso de los productos multimedia en España), REDCLN.com (Red de Centros de Negocio Local para la promoción del comercio electrónico), REGNET (la red de redes regionales para la publicación de datos del patrimonio histórico cultural), T3PYME (transferencia de tecnología telemática a PYMES), TIC como gestión del conocimiento (diagnóstico sobre la gestión del conocimiento en las empresas y su implementación por medio de las TIC); así como la creación del Centro Andaluz de Innovación y Tecnologías de la Información y las Comunicaciones (CITIC) con el objetivo de promover el desarrollo de las TIC.

23. Esta actuación consiste en proporcionar ayudas a la pymes industriales de menos de 10 empleados, para la incorporación de nuevas tecnologías, la innovación tecnológica y el comercio electrónico.

24. Mejorar la competitividad en las empresas en lo referente a la innovación y al desarrollo tecnológico.

25. Enmarcado dentro de la iniciativa comunitaria Leonardo da Vinci y el Programa Andaluz para el Fomento de la Economía Social, tiene como objetivo el fomento del empleo de los jóvenes dentro del ámbito de las nuevas tecnologías.

26. Proyecto de apoyo a los profesionales de la formación. Se inició en 1997 con los objetivos de crear un espacio para la teleformación, material multimedia interactivo para uso formativo, creación de una Red Andaluza de Profesionales de la Formación, etc. 
Por parte de la Consejería de Presidencia se vienen desarrollando una serie de iniciativas: con carácter general el Plan Estratégico para la SI Info@ landalus (2002-2004), y a partir de él las acciones encaminadas a la difusión de la SIC:

$1^{\circ}$ barómetro Info@landalus: estudio sobre el estado de la SI en Andalucía, desarrollando una serie de indicadores de medición.

Iniciativa Guadalinfo: aplicación a todos los sistemas informáticos de la Junta de Andalucía de software libre utilizando el sistema operativo Linux.

Portal Andaluciajunta.es: nuevo portal en Internet donde se reúne temáticamente información de interés de la Junta de Andalucía.

Debate en red: $2^{a}$ Modernización de Andalucía: espacio web (www.moderniza-dos. andaluciajunta.es) para el debate y foro público sobre temas de la SI en Andalucía y en relación a la evolución del plan Info@alandalus.

Desde otras consejerías de la Junta se están llevando a cabo diversas acciones impulsoras de la SI en la región, como es el caso de la Consejería de Educación y Ciencia con la Red Averroes o el Centro Informático Científico de Andalucía (CICA); la Consejería de Trabajo e Industria con el Plan de Internacionalización de la Empresa Andaluza, que incluye dentro de sus líneas de actuación el uso estratégico y de gestión en la empresa de las nuevas tecnologías; la Consejería de Economía y Hacienda con las Técnicas de Internacionalización de la empresa on line ${ }^{27}$; o la Consejería de Salud con la oficina virtual del Sistema Sanitario Público de Andalucía Inters@ @ , que permite a través de Internet realizar consultas, la gestión on line o la descarga de formularios de solicitud.

\section{REFLEXIONES Y CONSIDERACIONES FINALES}

El aumento de la competitividad se ha convertido en un requisito ineludible para las empresas en el actual contexto económico, caracterizado por la "imparable" globalización de los mercados, con la consecuente intensificación de la competencia, los incesantes avances en las tecnologías, el aumento de las exigencias de los consumidores, etc.

No cabe duda de que la generación y asimilación de innovaciones es uno de los principales factores que ha hecho posible la introducción del cambio en la empresa y el mantenimiento de la competitividad. Las empresas pueden incorporar la innovación de muy diversas formas, teniendo como objetivo el aumento en la calidad de sus productos o servicios, la disminución de costes, la mayor rapidez en su introducción en el mercado o la oferta de una mayor gama de productos o servicios.

27. Formación sobre la aplicación de las nuevas tecnologías dirigida a PYMES a través del organismo público Comercializadora de Productos Andaluces. 
Los principales obstáculos y rigideces que están impidiendo el correcto desarrollo de la innovación y la tecnología en nuestra región, se resumen entre otros en una escasa cultura innovadora y de cooperación entre empresas; escasa capacidad de la pymes para lanzar nuevos productos y servicios al mercado; insuficientes titulados en las empresas, sobre todo en las pymes, y escasez de técnicos y operarios expertos provenientes de la formación profesional; una investigación academicista, poco orientada a los problemas regionales; escasos espacios de innovación (Parques, Incubadoras, etc.), etc.

Por lo que respecta al capital humano, hemos comentado el importante avance del sistema educativo en Andalucía y el acortamiento de la distancia respecto a la media nacional. Sin embargo, también hemos apuntado la falta de adecuación entre las cualificaciones formativas y los requerimientos del mercado laboral. Y es que el sistema educativo ha de estar vinculado en todos sus niveles a la formación permanente, tanto de los jóvenes como de los demás ciudadanos.

Para que las redes de conocimiento tengan éxito en apoyar la innovación, además de la existencia de parques tecnológicos de última generación es necesario que los contactos entre la universidad y la industria sean constructivos en toda la región.

Como han demostrado diversos expertos en el tema, una de las funciones de las empresas de servicios avanzados a empresas es la de facilitar o propiciar la innovación en las empresas que demandan sus servicios. Pero, como hemos podido ver a partir de los datos que hemos presentado, las empresas que ofrecen estos servicios, empiezan por hacer ellas mismas un uso escaso de la innovación. Sin embargo, muchas de estas empresas ofrecen una gama de servicios a través de los cuales se promueve la innovación en sus clientes. La cuestión está en conseguir una mayor demanda de estos servicios entre las PYMES andaluzas.

La Administración andaluza está realizando importantes esfuerzos en instrumentar los mecanismos necesarios para fomentar y potenciar el avance de las TIC en nuestra región y el desarrollo de procesos de innovación entre las empresas andaluzas. El éxito de las políticas que se lleven a cabo dependerá, entre otros, de su capacidad para transformar los recursos económicos, científicos, sociales, culturales, históricos y territoriales en ventajas competitivas para nuestra región dentro del contexto de la globalización. 


\section{BIBLIOGRAFÍA}

ALBURQUERQUE, F. ET ALIA (2001), "Aprendiendo a innovar: el caso de Andalucía" en ROMÁN, C. (Ed.) (2001), páginas 61-133.

ANTONELLI, C. (2000), "New Information Technology and Localized Technological Change in the Knowledge-Based Economy", en BODEN, M. \& MILES, I. (eds.) Services and the Knowledge-Based Economy, Contiuum, Londres.

ASIÁN, R. (2000), "The Andalusian culture and its implications in the evolution of the service activities: is the entrepreneurial culture a determining factor in the present condition of the advanced business services in Andalusia?", X Conferencia Internacional de RESER, en Bergen los días 6 y 7 de Octubre.

ASIÁN, R. (2002), La Globalización de los Servicios en la Economía Andaluza. El Caso de los Servicios Avanzados a las Empresas. Tesis Doctoral. Sevilla.

ASIÁN, R. (2003a), "La oferta de servicios avanzados a las empresas en Andalucía en el contexto de la Nueva Economía" en Boletín Económico de Andalucía n 33-34: Nueva Economía y Sociedad del Conocimiento, páginas 219-232. Consejería de Economía y Hacienda, Junta de Andalucía.

ASIÁN, R. (2003b), “Andalucía ante los retos de la globalización. Los procesos de innovación y sus implicaciones en el desarrollo local”, V Congreso de la Asociación Andaluza de Ciencia Regional, Cádiz, Marzo 2003.

ASIÁN, R. y RODRÍGUEZ, J. (2003), “The role of knowledge systems and the innovation of ICT in regional development: analysis of the Andalusian region", IX Conference of Regional Studies Association, Pisa, Abril 2003.

BODEN, M. \& MILES, I. (2000), "Beyond the Services Economy", en BODEN, M. \& MILES, I. (eds.) Services and the Knowledge-Based Economy, Contiuum, Londres.

CAMACHO, R. (2001), "El desarrollo económico y social en Andalucía” en ROMÁN, C. (Ed.) (2001), páginas 49-59.

CAMACHO, J.A., RODRÍGUEZ, M. Y GARCÍA, R.M., (2003), “Innovación regional en España: ¿Influyen los servicios intensivos en conocimiento en la existencia de disparidades regionales?" XXIX Reunión de Estudios Regionales. Santander, 27-28 Noviembre 2003.

CASTAÑO, C. Y ROMÁN, C. (Dir. Coord.) (2002); Andalucía ante la Sociedad de la Información. Consejo Económico y Social de Andalucía (CES), Junta de Andalucía.

COTEC (2001), Innovación Tecnológica. Ideas Básicas. Fundación Cotec para la Innovación Tecnológica.

COTEC (2001), Innovación en Servicios. Estudio n ${ }^{\circ}$ 19. Fundación Cotec para la Innovación Tecnológica

DELGADO, M. (1981), Dependencia y marginación de la economía andaluza. Monte de Piedad y Caja de Ahorros de Córdoba. Córdoba. (Resumen de su Tesis doctoral. Facultad de Ciencias Económicas de Málaga, 1980). 
FUNDACIÓN BBV. Renta Nacional de España y su distribución provincial. Serie Homogénea. Años 1955 a 1995 y avances 1996 a 1999. Fundación BBV.

HIRSCH, D. (2001), "Regiones que aprenden y capital cultural: el caso de Andalucía”, en ROMÁN, C. (Ed.) (2001), páginas 13-24.

HOWELLS, J. (2002), "Innovation, Consumption \& Services - Encapsulation and the Combinatorial Role of Services", XXII International Conference of RESER, Manchester, 26-27 Septiembre, 2002.

JORDÁ BORRELL, R. (1998), "Formación del Sistema Ciencia-Tecnología-Industria", en Economía Andaluza (coordinador, J. Vallés Ferrer). Algaida editores, Sevilla.

ROMÁN, C. (1987), Sobre el Desarrollo Económico de Andalucía. Ed. Arguval, Málaga.

ROMÁN, C. (Ed.) (2001), Aprendiendo a innovar: Regiones del conocimiento. OCDE/IDR.

ROMERA, F. (2001), "Influencia de los Parques Científicos y Tecnológicos en su entorno local: el caso del Parque Tecnológico de Andalucía” en ROMÁN, C. (Ed.) (2001), páginas 167-187.

SASSEN, S. (1998), "Ciudades en la economía global: enfoques teóricos y metodológicos". Revista Eure, Vol.XXIV, nº 71, páginas 5-25. 